\title{
A RELATION BETWEEN NON-ALTERNATING AND INTERIOR TRANSFORMATIONS ${ }^{1}$
}

\author{
G. T. WHYBURN
}

Recently in proving certain existence theorems for non-alternating and for interior transformations of a continuum onto a simple $\operatorname{arc}^{2}$ it was observed that when a transformation of one of these types was set up, usually it satisfied in large measure conditions which brought it also under transformations of the other type. This suggests the existence of common ground shared by these sorts of transformations, and it is the object of this paper to exhibit the nature of such. Our principal conclusion is to the effect that, under certain auxiliary conditions, any interior transformation $f(M)=D$ of a compact continuum $M$ onto a dendrite $D$ can be factored into the form $f=f_{2} f_{1}$ where $f_{1}(M)=M^{\prime}$ merely shrinks sets of type $f^{-1}(p)$ ( $p$ an end point of $D$ ) in to single points and is topological elsewhere and $f_{2}\left(M^{\prime}\right)=D$ is nonalternating and interior. However, we first prove two theorems on the relation between the non-alternating property of a transformation of a continuum into a dendrite and the connectedness of the inverse sets for the end points of the dendrite.

THEOREM 1. If $M$ is a compact metric continuum and $f(M)=N$ is non-alternating, then for any end point $t^{3} p$ of $N, f^{-1}(p)$ is connected.

Proof. Suppose $f^{-1}(p)$ is not connected. Then there exist points $a$ and $b$ of $f^{-1}(p)$, a closed set $F \subset M-f^{-1}(p)$ and a separation $M-F=M_{a}+M_{b}$ where $a$ \& $M_{a}, b$ \& $M_{b}$. Since $f(F) \cdot p=0, f(F)$ is compact, and $p$ is an end point, there exists a neighborhood $U$ of $p$ in $N$ with $\bar{U} \cdot f(F)=0$ and such that the boundary of $U$ is a single point $q$. Since $q$ separates $p$ and $f(F)$ in $N$, it follows by a result of the author's ${ }^{4}$ that there exists a separation $M-f^{-1}(q)=M_{F}+M_{p}$, where $F \subset M_{F}, f^{-1}(p) \subset M_{p}$. But this gives at once the separation $M-f^{-1}(q)$ $=M_{a} \cdot M_{p}+\left(M_{F}+M_{b} \cdot M_{p}\right)$ where $M_{a} \cdot M_{p} \supset a,\left(M_{F}+M_{b} \cdot M_{p}\right) \supset b$, contrary to the hypothesis that $f$ is non-alternating.

THEOREM 2. If $f(M)=D$ is an interior transformation of a compact

${ }^{1}$ Presented to the Society, September 8, 1939.

${ }^{2}$ See my papers The existence of certain transformations, Duke Mathematical Journal, vol. 5 (1939), pp. 647-655; and Non-alternating interior retracting transformations, Annals of Mathematics, (2), vol. 40 (1939), pp. 914-921.

${ }^{3}$ That is, a point of Menger-Urysohn order 1 of $N$.

${ }^{4}$ See my paper in the American Journal of Mathematics, vol. 56 (1934), pp. 294$302,(1.4)$. 
continuum $M$ onto a dendrite $D$ such that for each end point $p$ of $D$, $f^{-1}(p)$ is connected, then $f$ is non-alternating.

Proof. Let $x, y \in D$ and let $R$ be the component of $D-x$ containing $y$. Then $R$ contains at least one end point $p$ of $D$. Since $f^{-1}(p)$ is connected, there exists a single component $Q$ of $M-f^{-1}(x)$ containing $f^{-1}(p)$. Since, ${ }^{5}$ by the interiority of $f$, any quasi-component of $f^{-1}(R)$ maps onto all of $R$ and hence intersects $f^{-1}(p)$, we have $Q=f^{-1}(R)$, whence $Q \supset f^{-1}(y)$ and thus $f$ is non-alternating.

COROLLARY. In order that an interior transformation $f(M)=D$ of a compact continuum $M$ onto a dendrite $D$ be non-alternating it is necessary and sufficient that for each end point $p$ of $D, f^{-1}(p)$ be connected.

TheOREM 3. Let $M$ be a compact continuum, let $D$ be a dendrite and let $H$ be the set of all end points of $D$. Any interior transformation $f(M)=D$ such that the collection $\left[f^{-1}(p)\right], p \varepsilon H$, is semi-closed ${ }^{6}$ can be factored into the form $f=f_{2} f_{1}$, where $f_{1}$ is equivalent to $f$ on $f^{-1}(H)$ and topological on $M-f^{-1}(H)$ and $f_{2}$ is interior and non-alternating.

Proof. Let us decompose $M$ into the sets $f^{-1}(p), p \varepsilon H$, and individual points of $M-f^{-1}(H)$. Since the collection $\left[f^{-1}(p)\right], p \varepsilon H$, is semiclosed, it follows by a result of the author' ${ }^{6}$ that this decomposition is upper semi-continuous. ${ }^{7}$ Let $M^{\prime}$ be the hyperspace of this decomposition and $f_{1}(M)=M^{\prime}$ the associated continuous transformation.

Now for each $x^{\prime} \varepsilon M^{\prime}$, let us define $f_{2}\left(x^{\prime}\right)=f f_{1}^{-1}\left(x^{\prime}\right)$. Then clearly $f(x) \equiv f_{2} f_{1}(x)$ for $x \varepsilon M$. The continuity of $f_{2}$ results at once from that of $f_{1}$ and $f$. Furthermore, since $f$ is interior it follows ${ }^{8}$ that $f_{2}$ is interior. Finally, since for any end point $p$ of $D$ we have that $f_{2}^{-1}(p)=f_{1} f^{-1}(p)$ is a single point of $M^{\prime}$, it follows by Theorem 2 that $f_{2}$ is non-alternating. This completes the proof of our theorem.

Since any finite collection of disjoint closed sets is semi-closed, we have the following corollary:

Corollary. Any interior transformation of a compact continuum into a simple arc (or into a dendrite with only a finite number of end points) can be factored into the form described in Theorem 3.

UNIVERSITY OF VIRGINIA

5 See my paper, Duke Mathematical Journal, vol. 3 (1937), pp. 370-381, (1.4).

${ }^{6}$ That is, any convergent sequence of the sets $\left[f^{-1}(p)\right]$ whose limit set intersects $M-f^{-1}(H)$ converges to a single point. See my paper in the Duke Mathematical Journal, vol. 2 (1937), pp. 685-690.

${ }^{7}$ See R. L. Moore, Foundations of Point Set Theory, American Mathematical Society Colloquium Publications, vol. 13, 1932, chap. 5.

${ }^{8}$ See result (1.6) of the paper cited in footnote 5. 